\title{
Comment on the Paper by Cauwenberghs
} and Kuznetsova Entitled 'Determinants and Prognostic Significance of the Renal Resistive Index'

\author{
Yuhei Kawano Yoshio Iwashima \\ a Division of Medical Technology, Teikyo University Fukuoka, Omuta, and ${ }^{b}$ Division of \\ Hypertension and Nephrology, National Cerebral and Cardiovascular Center, Suita, Japan
}

The clinical use of Renal Doppler ultrasonography is spreading widely because it can assess renal blood flow in a non-invasive evaluation. It is recommended as a screening examination for patients with suspected renovascular hypertension [1]. The renal resistive index (RRI) derived from intrarenal Doppler waveforms has also been drawing attention. In this issue of Pulse, Cauwenberghs and Kuznetsova [2] presented a review article that focused on the RRI with particular reference to its determinants and prognostic significance. We would like to make some comments about the clinical meanings of the RRI and the article by Cauwenberghs and Kuznetsova [2].

\section{Determinants of the RRI}

The RRI is derived from intrarenal Doppler waveforms as [(peak systolic velocity - enddiastolic velocity)/peak systolic velocity] at the renal segmental artery. It seems to reflect intrarenal hemodynamics and the renal vascular pathological process. However, neither precise quantitative measures of the regional renal blood flow nor the level of regional blood pressure can be obtained by renal Doppler ultrasonography. It has been shown that several factors, such as systemic hemodynamics, peripheral vascular resistance and compliance, heart rate, and renal interstitial pressure, affect the RRI [2-7]. Therefore, intrarenal hemodynamics and renal vascular properties are not the exclusive determinants of the RRI. As Cauwenberghs and Kuznetsova [2] described in their article, the RRI is a product of complex interaction between renal and systemic vascular wall properties and hemodynamic factors. Several anthropometric factors, such as body weight and height, have also been shown to be independent determinants of RRI. 
Several studies have demonstrated a significant and direct association between RRI and central or peripheral pulse pressure [6-8]. The main determinant of the pulsatile component of pulse pressure is compliance of large arteries, and wide pulse pressure has been linked to poor cardiovascular outcome independent of mean blood pressure. O'Rourke and Safar [9] suggested that increased pulsatile stress causes endothelial and muscular damages in small arteries of the brain and the kidney. Because RRI reflects the pulsatility of renal arteries, it might be useful for the early detection of renal microvascular damage [2].

\section{Prognostic Significance of the RRI}

It has been shown that the RRI is associated to the status or prognosis of various renal pathological conditions. Those conditions include renal allograft rejection, renal artery stenosis, chronic kidney disease, critically ill patients, and essential hypertension [2]. Although the RRI is not a specific determinant of renal hemodynamics and vascular wall properties, it seems to be a useful prognostic marker in some conditions. Radermacher et al. [10] have shown that the RRI is useful to predict the outcome of renal arterial angioplasty. They also reported that the RRI was associated with the progression of chronic kidney disease [11].

In a previous study, we investigated the prognostic role of the RRI in cardiovascular and renal outcomes in 426 patients with essential hypertension without previous cardiovascular disease [12]. In multivariate Cox regression analysis, the RRI was an independent predictor of worse outcome not only in total subjects but also in patients with chronic kidney disease. In our study, the combination of high RRI and low estimated glomerular filtration rate was a powerful predictor of cardiovascular and renal outcomes. In another study, we also investigated the association of the RRI with target organ damage, namely carotid atherosclerosis, left ventricular hypertrophy, and albuminuria, in 288 patients with essential hypertension [13]. In multiple logistic regression analysis, each standard deviation increase in the RRI gave $47 \%$ higher odds of having left ventricular hypertrophy and $70 \%$ higher odds of having albuminuria. Our studies suggest that an increased RRI is a marker of subclinical target organ damage as well as a predictor of cardiovascular and renal outcomes in essential hypertension. Another recent study validated the independent association of the RRI with allcause mortality in patients with chronic kidney disease [14].

Therefore, the RRI may provide valuable information in the assessment of intrarenal hemodynamics, subclinical organ damage, and future cardiovascular and renal outcomes. However, we have to be aware of its limitations, particularly the fact that the RRI is affected by several factors other than the kidney, such as systemic hemodynamics. Although there are many studies about the RRI, investigations focusing on its prognostic role are still limited. Further investigation will be required in this area to provide conclusive evidence to guide clinical practice.

\section{Disclosure Statement}

The authors have no conflicts of interest to declare. 
Kawano and Iwashima: Comment on the Paper by Cauwenberghs and Kuznetsova Entitled 'Determinants and Prognostic Significance of the Renal Resistive Index'

\section{References}

1 Shimamoto K, Ando K, Fujita T, Hasebe N, Higaki J, Horiuchi M, et al: The Japanese Society of Hypertension Guidelines for the Management of Hypertension (JSH 2014). Hypertens Res 2014;37:253-392.

2 Cauwenberghs N, Kuznetsova T: Determinants and prognostic significance of renal resistive index. Pulse 2015;3:172-178.

3 Tubin ME, Bude RO, Platt JF: The resistive index in renal Doppler sonography. Where do we stand? AJR Am J Roentgenol 2003;180:885-892.

4 Bude RO, Rubin JM: Relationship between the resistive index and vascular compliance and resistance. Radiology 1999;211:411-417.

5 Gottlieb RH, Luhmann K, Oates RP: Duplex ultrasound evaluation of normal native kidneys and native kidneys with urinary tract obstruction. J Ultrasound Med 1989;8:609-611.

6 Ponte B, Pruijm M, Ackermann D, Vuisteiner P, Eisenberger U, Guessous I, et al: Reference values and factors associated with renal resistive index in a family-based population study. Hypertension 2014;63:136-142.

7 Kuznetsova T, Cauwenberghs N, Knez J, Thijs L, Liu YP, Gu YM, et al: Doppler indexes of left ventricular systolic and diastolic flow and central pulse pressure in relation to renal resistive index. J Hypertens 2015;28:535545.

8 Hashimoto J, Ito S: central pulse pressure and aortic stiffness determine renal hemodynamics: pathophysiological implication for microalbuminuria in hypertension. Hypertension 2011;58:839-846.

9 O’Rourke MF, Safar ME: Relationship between aortic stiffening and microvascular disease in brain and kidney: cause and logic of therapy. Hypertension 2005;46:200-204.

10 Radermacher J, Chavan A, Bleck J, Vitzhum A, Stoess B, Gebel MJ, et al: Use of Doppler ultrasonography to predict the outcome of therapy for renal artery stenosis. N Engl J Med 2001;344:410-417.

11 Radermacher J, Ellis S, Haller H: Renal resistance index and progression of renal disease. Hypertension 2002; 23:699-703.

12 Doi Y, Iwashima Y, Yoshihara F, Kamide K, Hayashi S, Kubota Y, et al: Renal resistive index and cardiovascular and renal outcomes in essential hypertension. Hypertension 2012;60:770-777.

13 Doi Y, Iwashima Y, Yoshihara F, Kamide K, Takata H, Fujii T, et al: Association of renal resistive index with target organ damage in essential hypertension. Am J Hypertens 2012;25:1292-1298.

14 Toledo C, Thomas G, Schold JD, Arrigain S, Gornik HL, Nally JV, et al: Renal resistive index and mortality in chronic kidney disease. Hypertension 2015;66:382-388. 